\title{
VERGÜTUNGSSYSTEME
}

\section{Leistung soll sich lohnen}

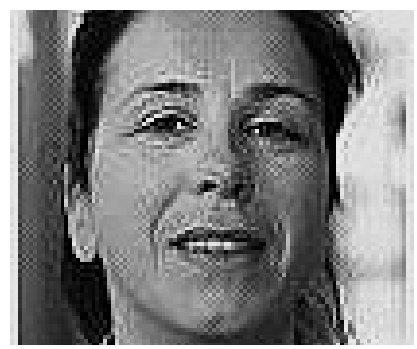

VON GABRIELE MOOS

Prof. Dr. Gabriele Moos leitet den Studiengang Gesundheits- und Sozialwirtschaft am RheinAhrCampus in Remagen.

E-Mail

moos@rheinahrcampus.de

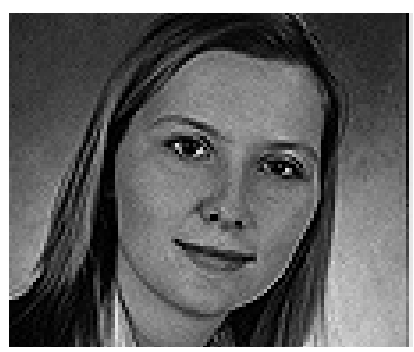

SARA BODE

Sara Bode ist Personal- und

Organisationsberaterin bei der contec $\mathrm{GmbH}$ in Stuttgart.

E-Mail bode@contec.de

\author{
Das heikle Thema der Entlohnung von Führungskräften \\ beschäftigt auch sozialwirtschaftliche Organisationen \\ und Unternehmen. Eine neue Vergütungsstudie sorgt \\ nun für eine bessere Datenbasis.
}

Die Höhe des Gehaltes ist zwar nicht alleine ausschlaggebend für die Wahl eines Arbeitgebers, aber es birgt erhebliches (De-) Motivationspotential. Um dieses Unternehmensrisiko, das sich mit dem aufkommenden Fach- und Führungskräftemangel noch verschärft, einzudämmen, bedarf es eines neuen Umgangs mit dem Thema »Vergütungsstrukturen und Leistungsanreize .

Ziel einer gelungenen Vergütungssystematik ist es, für beide Seiten - Unternehmen und Mitarbeitende - eine akzeptable Lösung zu finden: Für den Arbeitgeber muss das System finanzierbar sein, Mitarbeitende möchten fair entlohnt werden. Beiden Parteien ist es gleichermaßen wichtig, dass das Gehalt marktüblich und angemessen ist. Dies ist aber auch aufgrund fehlender Transparenz der Gehaltsstrukturen in der Sozialwirtschaft in Moment kaum möglich. Aus diesem Grund hinterlassen Gehaltsverhandlungen oftmals auf beiden Seiten ein ungutes Gefühl.

Die aktuelle wissenschaftliche Studie der contec GmbH in Zusammenarbeit mit dem RheinAhr-Campus in Remagen (1) zeigt, dass derzeit knapp die Hälfte der Führungskräfte mit ihrem Gehalt unzufrieden sind. An der Studie teilgenommen haben 358 hauptamtliche Führungskräfte wie Vorstände, Geschäftsleitungen, Regional- und Bereichsleitungen aus der Sozialwirtschaft.

Bezogen auf die berufliche Position im Verhältnis zum Gehalt hat sich gezeigt, dass die Gehaltshöhe mit der üb- lichen Hierarchie sozialwirtschaftlicher Unternehmen übereinstimmt. Somit verdienen die befragten Vorstände mit durchschnittlich 84.807 Euro deutlich mehr als Geschäftsleitung mit durchschnittlich 75.144 Euro. Das geringste Durchschnittseinkommen erreicht die Einrichtungsleitung mit 47.421 Euro.

So aufschlussreich die Kenntnis der reinen marktüblichen Vergütung ist, so wichtig ist es, ob die Befragten die IstSituation als angemessen empfinden. Knapp die Hälfte aller Teilnehmer erachtet ihre derzeitige Vergütung als nicht angemessen (vgl. Tabelle). Im Durchschnitt empfinden sie eine Erhöhung um etwa 20 Prozent, ausgehend vom Durchschnittsgehalt ihrer Gruppe, als richtig. Besonders die hierarchisch hohen Positionen (Vorstand und Geschäftsleitung) und die Verwaltungsleitungen empfinden Ihre Gehaltshöhe als nicht ausreichend.

Auch wenn viele Führungskräfte insbesondere im Vergleich zur freien Wirtschaft - niedriger vergütet werden, ist doch fraglich, ob die Unternehmen die Mehrbelastung des als angemessen erachteten Gehaltes tragen können. Weiterhin ist zweifelhaft, ob dadurch eine dauerhafte Motivations- und Leistungssteigerung erzeugt wird.

Folgt man der Zwei-Faktoren-Theorie (2) des Arbeitswissenschaftlers und klinischen Psychologen Frederick Herzberg, gehört das Gehalt zu den Punkten, die zwar Unzufriedenheit verhindern können, nicht aber an sich zu einer Zufriedenheit führen. Dies kann viel mehr über Anerkennung von geleis- 
teter Arbeit, Leistungserfolge, Selbstständigkeit, Arbeitsaufgaben und Arbeitsinhalte hervorgerufen werden.

Aber auch das Gehalt kann eine Rückmeldung und somit Anerkennung der geleisteten Arbeit sein. Die traditionellen Entgeltsysteme mit ausschließlichem Fixgehalt beziehen dabei weder individuellen Leistungen noch den aktuellen wirtschaftlichen Erfolg ein und werden somit zunehmend als leistungshemmend, demotivierend und zu teuer empfunden.

Bei der Frage nach der Anreizwirkung zur Leistungssteigerung von variabler Vergütung gaben insgesamt über ein Drittel der Teilnehmenden der Vergütungsstudie an persönlich einen starken oder sehr starken Anreiz zu sehen. Auf die Frage, inwiefern die Erhöhung des variablen Gehaltsbestandteils ihr persönliches Verhalten beeinflusse, gaben knapp 20 Prozent der Führungskräfte an, dass sich ihre Arbeitsmotivation stark verändern würde. Bei 6,6 Prozent würde sich das Arbeitsverhalten und bei 8,9 Prozent ihre Arbeitsleistung stark verändern. Besonders auffällig ist, dass 34,5 Prozent angeben, ihr Arbeitsverhalten durch eine Erhöhung des variablen Ge- haltsanteils stark bzw. etwas zu verändern. Sogar 36,9 Prozent geben an, dass sich ihre Arbeitsleistung stark bzw. etwas durch eine solche Erhöhung verändern würde.

Auf dieser Grundlage lässt sich schlussfolgern: Eine Erhöhung des variablen Gehaltsanteils hätte nicht nur Auswirkungen auf die Motivation der Führungskräfte, sondern auch eine direkte qualitative und quantitative Leistungsveränderung zur Folge.

Damit aber ein variables Entgeltsystem nachhaltig funktionieren kann, müssen die Bewertungskriterien von allen Beteiligten akzeptiert werden. Von den Führungskräften selbst wird beispielsweise der Zielerreichungsgrad, die Kundenzufriedenheit oder der Auslastungsgrad als zweckmäßig angesehen. Mögliche weitere Kriterien sind die Umsatzrentabilität, der Innovationsgrad, die Liquidität oder der Erfolg bei fachlichen Projekten. Entscheidend für das Verständnis ist hierbei die Passgenauigkeit zur Position und dass die Hintergründe für Vorgaben wie Umsatzgrößen transparent kommuniziert werden.

Egal, ob die Vergütung mittels Festgehalt oder mit einem variablen Entgel- tanteil gestaltet ist, die transparente und nachvollziehbare Gestaltung muss die Grundlage sein. Hierzu gilt es sowohl die branchenüblichen Gehälter, das vorhandene Gehaltsgefüge, aber auch die individuelle Unternehmenssituation einbezogen werden.

Nicht vernachlässigt werden darf die Auswirkung einer wertschätzenden Unternehmenskultur, in der Fairness, Respekt und Vertrauen eine tragende Rolle spielen. Denn erst das Zusammenspiel dieser monetären wie nicht monetären Leistungsanreize führen zur Zufriedenheit der Mitarbeitenden, sorgen für niedrigere Fluktuationsraten und sichern darüber hinaus die Wirtschaftlichkeit des Unternehmens.

\section{Anmerkungen}

(1) Weitere Informationen: Die Vergütungsstudie 2010 » Führungskräfte in der Sozialwirtschaft « kann zum Preis von 75,- Euro zzgl. Versand bei der contec GmbH (info@contec.de) bestellt werden.

(2) Vgl. Herzberg 1959, zitiert nach Berthel/ Becker (2007): Personal-Management. Grundzüge für Konzeptionen betrieblicher Personalarbeit, S. $23 \mathrm{f}$.

\begin{tabular}{|llll|}
\hline Position & Derzeitiges Gehalt & Unzufriedenheitsquote & Gewünschter Mehrbetrag \\
\hline Vorstand & $84.807 €$ & $43,6 \%$ & $24.557 €$ \\
\hline Geschäftsleitung & $75.144 €$ & $52,6 \%$ & $22.376 €$ \\
\hline Verwaltungsleitung & $65.191 €$ & $42,9 \%$ & $14.550 €$ \\
\hline Regionalleitung & $62.921 €$ & $14,3 \%$ & $16.779 €$ \\
\hline Bereichsleitung & $49.461 €$ & $40,0 \%$ & $14.300 €$ \\
\hline Einrichtungsleitung & $47.421 €$ & $38,8 \%$ & $10.201 €$ \\
\hline & & Führungskräfte in der Sozialwirtschaft sind zum großen Teil \\
& & mit ihrem Gehalt unzufrieden, wie die "Vergütungsstudie
\end{tabular}

